\title{
Automatic Cell Tracking Applied to Analysis of Cell Migration in Wound Healing Assay
}

\author{
Ryoma Bise, Takeo Kanade, Zhaozheng Yin, and Seung-il Huh
}

\begin{abstract}
The wound healing assay in vitro is widely used for research and discovery in biology and medicine. This assay allows for observing the healing process in vitro in which the cells on the edges of the artificial wound migrate toward the wound area. The influence of different culture conditions can be measured by observing the change in the size of the wound area. For further investigation, more detailed measurements of the cell behaviors are required. In this paper, we present an application of automatic cell tracking in phasecontrast microscopy images to wound healing assay. The cell behaviors under three different culture conditions have been analyzed. Our cell tracking system can track individual cells during the healing process and provide detailed spatio-temporal measurements of cell behaviors. The application demonstrates the effectiveness of automatic cell tracking for quantitative and detailed analysis of the cell behaviors in wound healing assay in vitro.
\end{abstract}

\section{INTRODUCTION}

Analysis of cell behaviors in population is important for research and discovery in biology and medicine. To effectively obtain the quantitative measurements of cell behaviors, automatic cell tracking system is important. Many tracking algorithms have been developed to correctly segment and track cells in populations. These existing tracking algorithmic papers which describe the algorithms are valuable for computer researchers to improve the tracking algorithms in order to achieve higher accuracy. To facilitate cell behavior analysis for biological researchers, it is also important to show how easily and effectively biological measurements can be obtained by applying tracking algorithms to biological experiments. Some papers presented biological measurements computed from tracking results. In [1], House et $a l$. introduced a set of measures of cell morphology and trajectories including displacement, path length, speed, and changes and persistence in movement direction to describe cell migration. In [2], Padfield et al. applied their tracking algorithms for fluorescent images and demonstrated how biological measurements (i.e, the number of mitosis/arrested cells, migration speed and mean intensity of cell areas) change over time in order to show that the tracking system is useful tool for biological research.

In this paper, we aim to show how easily and effectively automated cell tracking systems can provide detailed

This work was basically done when Ryoma Bise worked at Carnegie Mellon University. He currently works at Dai Nippon Printing Co., Ltd., Tokyo, Japan. bise-remail.dnp.co.jp

Takeo Kanade, Zhaozheng Yin, and Seung-il Huh are with the Robotics Institute, Carnegie Mellon University, Pittsburgh, PA, USA. tk@Cs.cmu.edu, yinz@cmu.edu, seungilh@cs. Cmu.edu

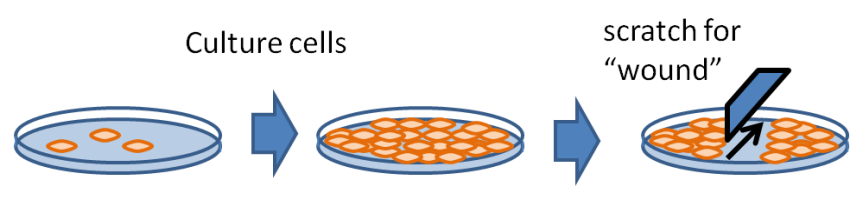

Fig. 1. The process of making wound. (a)
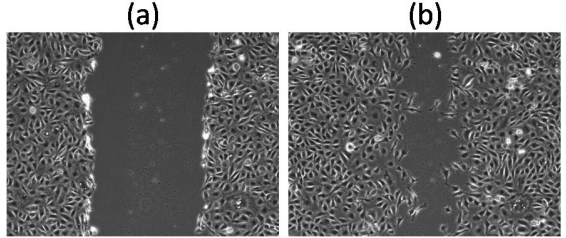

(c)

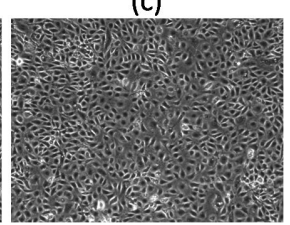

Fig. 2. Example images of the wound healing process. (a) the initial image on the healing process. (b) an image at which cells move to wound area. (c) an image at which cells fill the wound area.

spatio-temporal cell behavior measurements for biological analysis rather than discovering new biological phenomena and proposing new tracking algorithms. The spatio-temporal measurements of cell behaviors are important for critical analysis, because the cell culture conditions vary with time and space on the dish. For example, an effect of a medicine may change with time and space since cell density can be different on different spaces. We present an application of automatic cell tracking for wound healing assay in vitro under different culture conditions to show the effectiveness of cell tracking system for biological research.

The wound healing assay is an easy and low-cost method to allow for observing cell migration in vitro [3]. In this assay, cells are firstly grown to form a confluent monolayer in vitro. An artificial wound is generated by scratching and displacing a group of cells at the center as shown in Fig.1, and then the healing process is observed while neighboring cells fill in the wound area as shown in Fig.2 (a-c). This healing process takes 3 to 24 hours, depending on cell types and culture conditions. The healing process is monitored by a sequence of microscopic images. Liang et al. [3] compared several migration assays in vitro and described advantages of using the wound healing assay that mimics cell migration in vivo. For example, endothelial cells (ECs) in vitro mimic the process in which ECs in the blood vessels migrate into the denuded area to close the wound. Yarrow et al. [4] measured the healing speed by observing the size of the wound area in order to analyze the effectiveness of different culture conditions. For further analysis of the effectiveness 


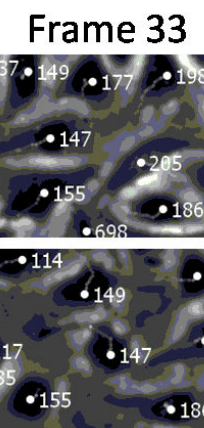

Frame 65
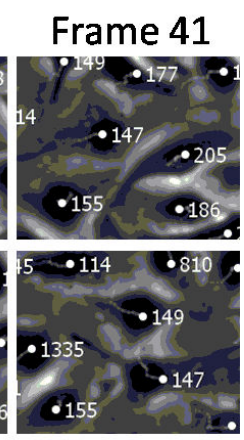

Frame 73
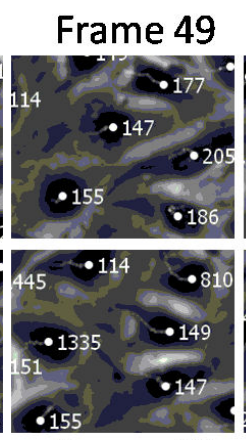

Frame 81

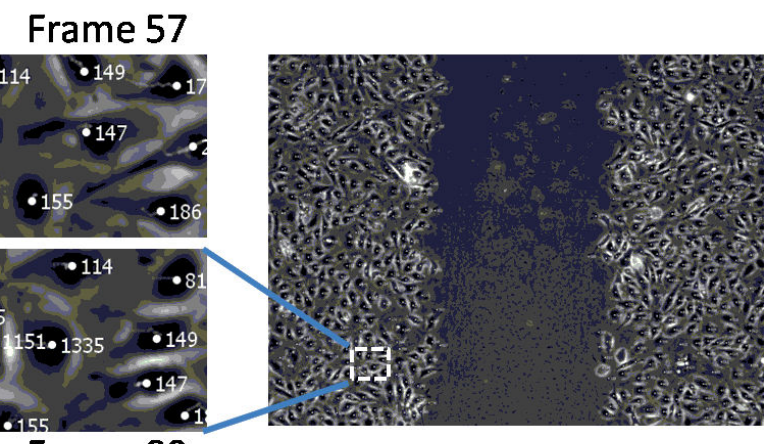

Frame 89

Fig. 3. Right: an example image of the tracking result. Left: an example image sequence of the zooming images that correspond with the white dot rectangle in the right image.

of the cell culture condtions, more detailed measurements of the cell behaviors are often required. For example, Abbi et $a l$. [5] analyzed the cell migration path to assess the effects of expression of exogenous genes on migration of individual cells. Nikolic et al. [6] manually tracked cell migration in wound healing assay in order to understand how multiple cells execute highly dynamic and coordinated movements during the healing process. Cell tracking allowed them to analyze how individual aspects of the wound contribute to the coordinated dynamics of cells.

We applied our automatic cell tracking method to sequences of phase-contrast microscopy images of a wound healing assay in vitro under three different culture conditions (i.e., three different amounts of medicine "Latrunculin B "that interferes with cellular activity). Our system can locate cell regions and track more than hundreds of cells individually under non-invasive imaging. It allows us to compute spatio-temporal measurements including the cell density, migration speed and direction, statistics of mitosis events, and their mutual dependency in order to analyze how the cell culture conditions (i.e., amount of the medicine) affect the cell behaviors over time and space. These measurements can provide critical information for investigating the healing process.

\section{Cell Tracking System}

To track cells, we have adopted our tracking method recently developed in [8]. In this section, we briefly introduce our tracking method.

\section{A. Cell Detection}

To facilitate segmentation, we have adopted the image restoration technique recently developed in [9]. The technique utilizes the optophysical principle of image formation by phase contrast microscope, and transforms an input image into an artifact free image by minimizing a regularized quadratic cost function. With artifacts removed, high quality segmentation can be achieved by simply thresholding the restored image.

\section{B. Mitosis Detection}

To detect the birth events, we have adopted the mitosis detection technique recently develped in [10]. Firstly, as mitosis events generally exhibit increase of brightness, bright regions are extracted as patches, and then candidate patch sequences are constructed by associating patches. Next, the gradient histogram features are extracted from the patches. Finally, a probabilistic model named Event Detection Conditional Random Field (EDCRF) is applied to determine whether each candidate contains a birth event and which frame the birth event is located in.

\section{Tracking Algorithm}

Based on the segmentation and mitosis detection results, we proposed a cell-blob association approach tolerant of missing cell detection, cell overlapping and dividing. The tracking system is automatically initialized by the segmentation algorithm and can handle objects coming into or leaving the field of view. Fig. 3 shows an example of the tracking result where the cells are well tracked in high confluence. The tracking system assigns a positive integer ID to each cell that is being tracked as its unique identifier. As its descriptor, each cell has its parent identifier Parent-ID for maintaining its lineage information (Parent-ID $=0$ for cells with no parent, i.e., those cells that appear in the first frame or enter to the field of view) and its state information (i.e., its centroid and contour shape of the cell region) at each frame. Using this information, various cell behavior measurements can be computed.

\section{Space-Time Analysis of Cell Behaviors of WOUND HEALING}

We applied our tracking system for experiments which aim to screen out cell migration inhibitors. Cell migration inhibitor is important for inhibiting the migration ability of cancer cells [11][12]. In general, the cell behaviors where the cells move toward to an open wound in a cellular mono-layer is thought to predict their migratory ability. Fig. 4 shows the overall flow of wound healing analysis experiment. Firstly, the culture dishes with wound area are prepared under difference types of culture conditions. These dishes are monitored 


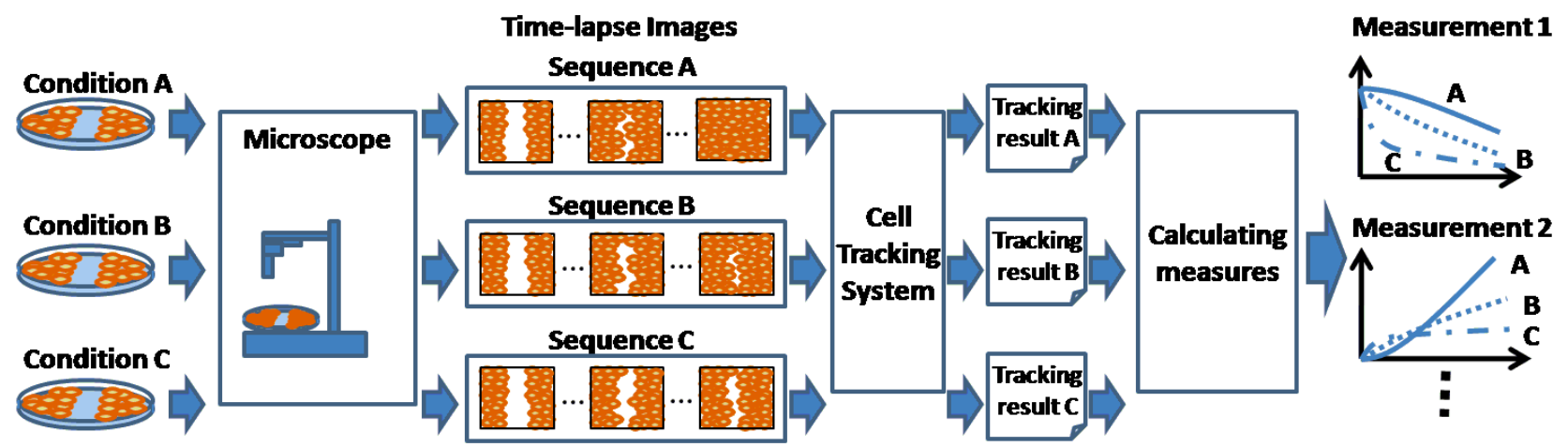

Fig. 4. Flow of our wound healing assay experiments under three culture conditions.

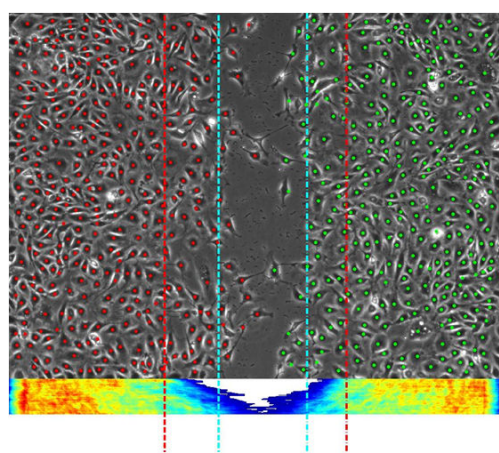

Fig. 5. The jet map of cell density changes over time with tracking results, each row of which represents the density. In the jet map, red color shows higher density, blue color shows lower density.

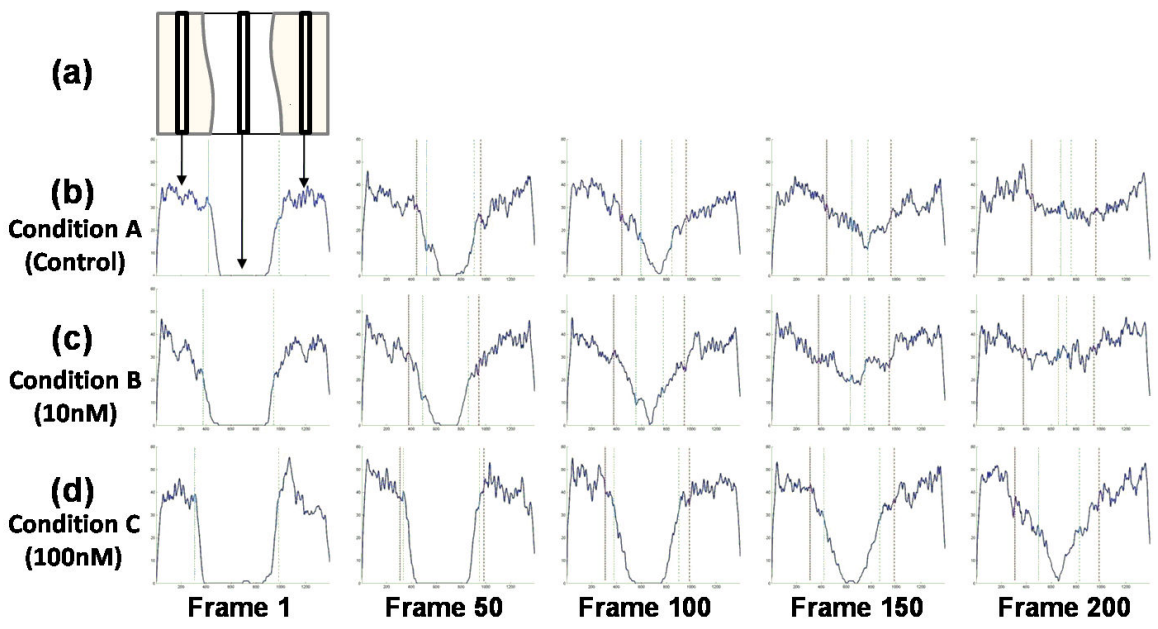

Fig. 6. The space-time transition of the cell density. by microscope, generating a time-lapse image sequence. The image sequences are inputted to the automatic cell tracking system. From the tracking results, various measurements that characterize the cell behavior are calculated. In the experiments, we compute the spatio-temporal measurements under three different culture conditions (i.e., three different amounts of medicine "Latrunculin B "that interferes with cellular activity) by using tracking results. It allows us to easily analyze how the cell behaviors change over time and location.

\section{A. Cell Culture Conditions and Imaging Modality}

The followings are how we prepared the cell culture dishes and how the image sequences were obtained for the experiments.

1) Cell Culture Conditions: On three dishes, BAEC (bovine aortic endothelial cells) were cultured under three different culture conditions. For each dish, a group of cells at the center of the dish was scratched and displaced on a confluent monolayer. Different amount of medicine was added to each dish.

Condition A : control (no medinine)

Condition B : 10nM (nano molar) of Latrunculin B

Condition $\mathrm{C}: 100 \mathrm{nM}$ of (nano molar) Latrunculin B
2) Time-lapse Imaging: The area around the wound area in each dish is monitored with a Leica DMI 6000B inverted microscope using a $10 \mathrm{X}$ objective with phase optics until neighboring cells fill completely. Images were acquired every 5 minutes for 17 hours using a 12-bit CCD camera with each image of $1040 \times 1392$ pixels.

\section{B. Cell Behavior Characteristic Measurements}

Using tracking results, we calculated various measurements of cell behavior characteristics. In cell behavior analysis, the change of the cell density over time on the whole area is a useful index [13][14]. To investigate how the cell culture conditions affect the cell migration, speed and direction of the cell migration are often measured [13][15]. Cell culture condition usually affects both migration and proliferation. To separate these affects, the statistics of the mitotic events are important. Our system allows us to compute all of these spatio-temporal behavior characteristics in detail, including cell density, the speed and the direction of cell migration, and the statistics of mitosis events.

1) Cell Density: Figs. 5 and 6 show how the cell density changes over time and location. Since cells generally migrate horizontally in the experiments as shown in Fig.2, the cell 


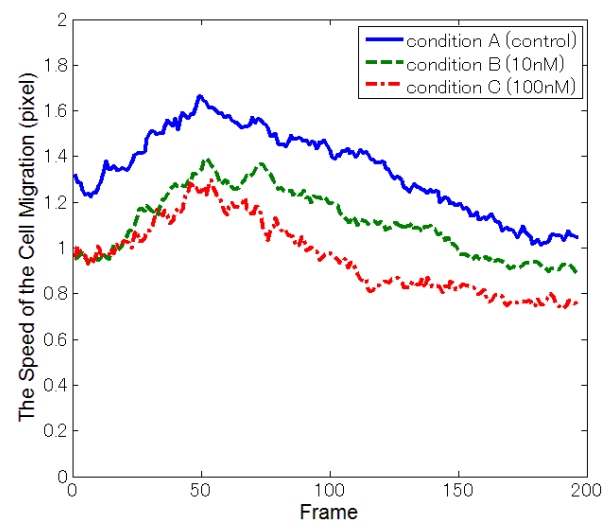

Fig. 7. The average speed of the cell migration over the time.

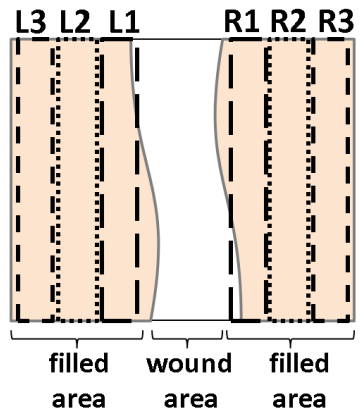

Fig. 8. Local areas.

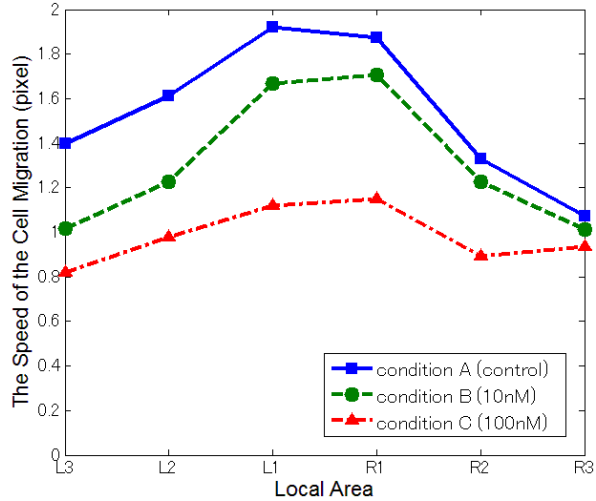

Fig. 9. The average speed of the cell migration at each local area.
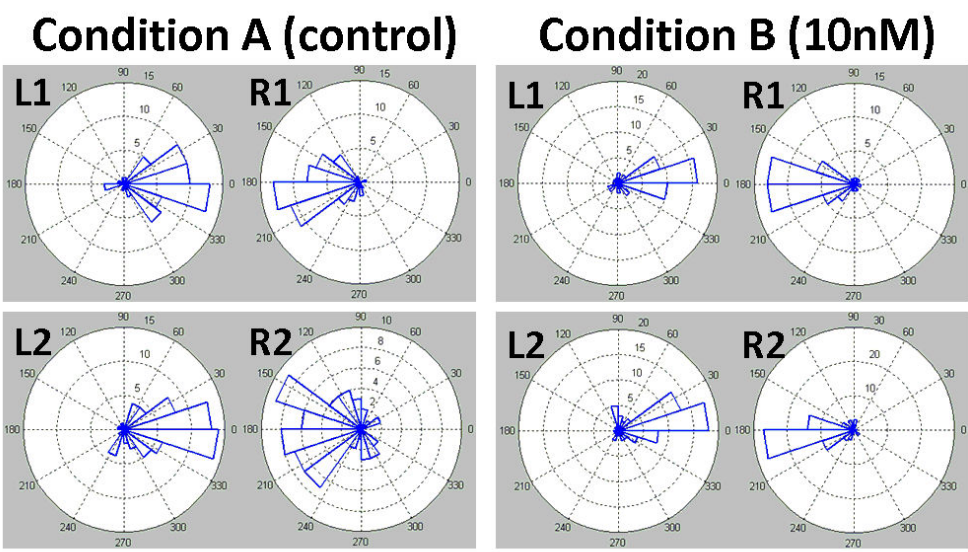

\section{Condition C (100nM)}
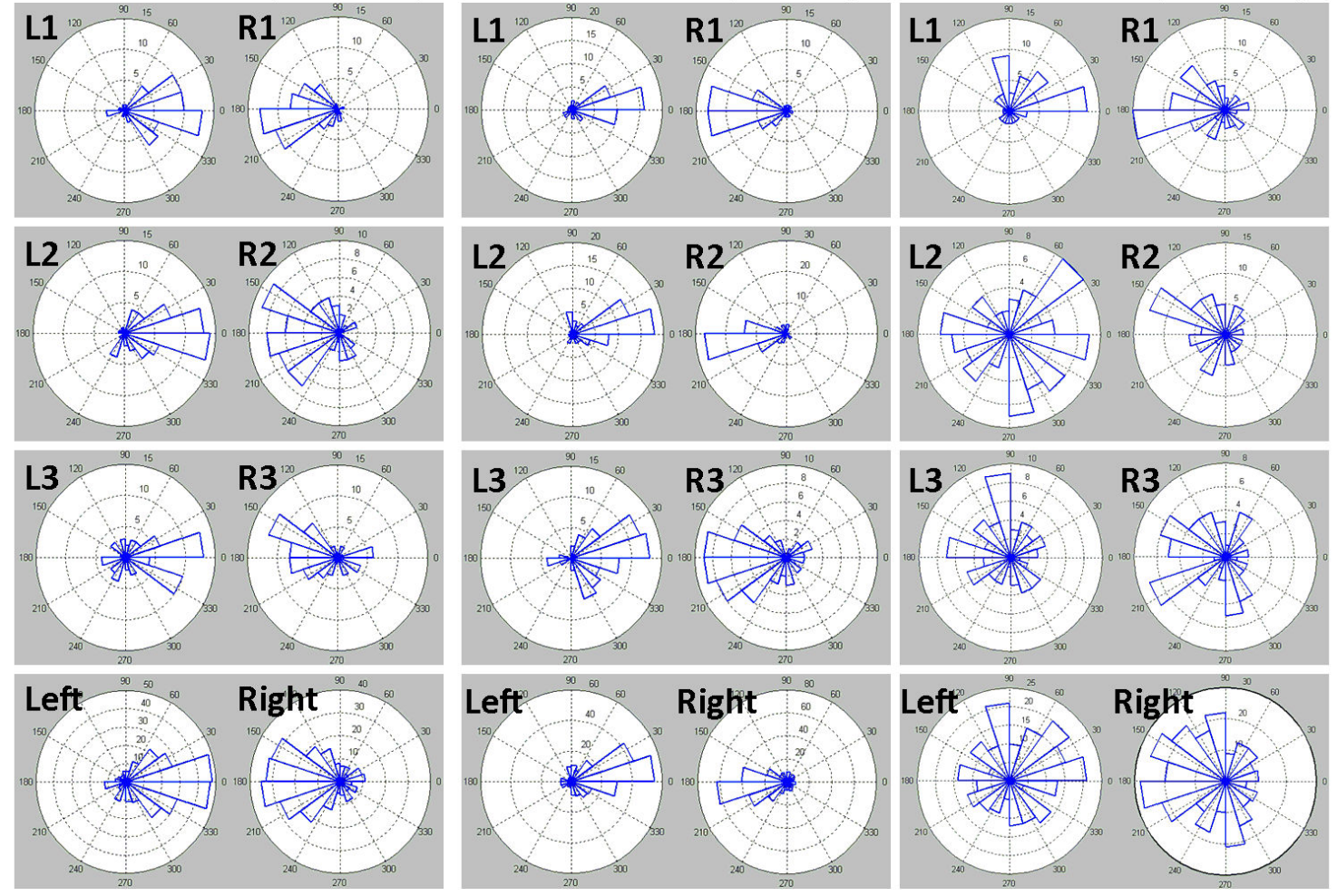
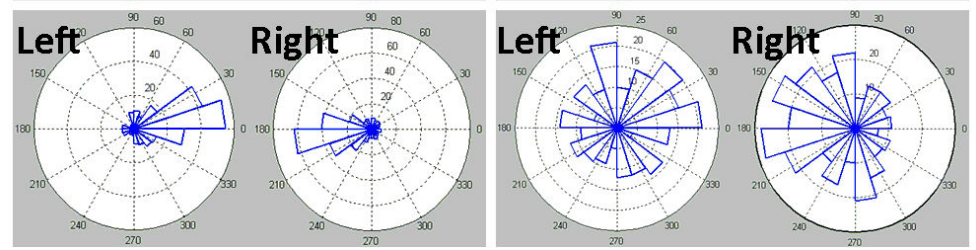

Fig. 10. Rose diagrams of cell migration directions on each local area.

density is computed over narrow vertical window (the width of the window is 40 pixels, i.e., $36.5 \mu \mathrm{m}$ ) as shown in Fig. $6(a)$. The vertical lines show that the $95 \%$ cell migration front of left and right sides, which is defined as the 95th percentile line of the total cell count of each side. Red color lines indicate the $95 \%$ line at the initial frame, blue dotted lines indicate the $95 \%$ line at the current frame. Fig. 6 (b-d) show the comparison of the space-time transition of the cell density under three different conditions. At the second row (condition A), the cell density in the wound area is low at frame 1 . Then, cells in the left and right regions migrate into the central area and the density in the wound area increases until it becomes flat in frame 200. We observe the similar behaviors for the other conditions, but cells on condition $\mathrm{C}$ $(100 \mathrm{nM})$ migrate more slowly than those in condition A and B. The density in the wound area is still low at the end of the sequence.

2) Speed of the Cell Migration: To analyze how the speed of cell migration changes over time, we computed the average speed of the cell migration over the whole area in each frame. The results presented in Fig. 7 show that the speed in the condition A (control) is consistently higher than those in other conditions, and the speed in the condition $\mathrm{C}$ $(100 \mathrm{nM})$ is the slowest. The migration speed firstly increases 


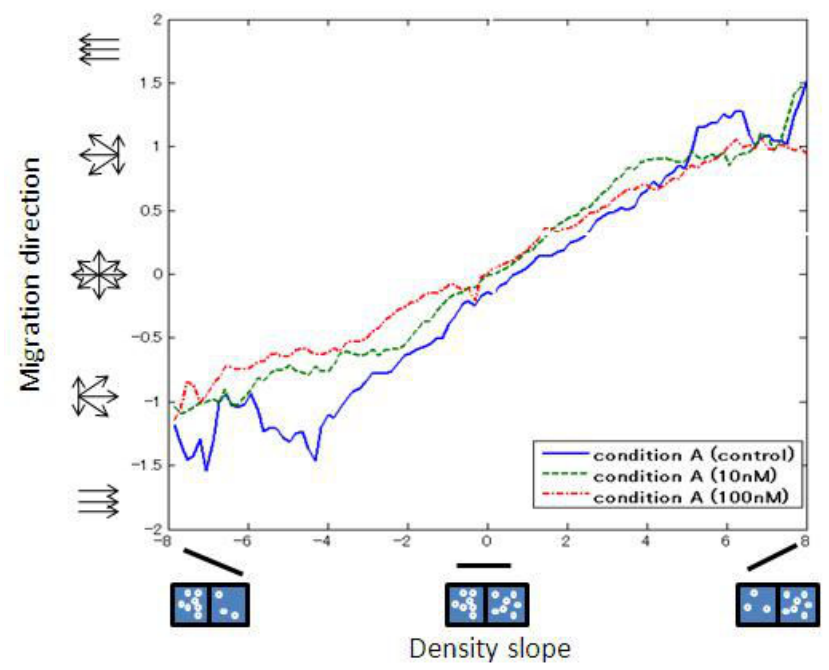

Fig. 11. The relationship between density slope and migration direction.

until frame 50, and then it continuously decrease with the time.

It is conceivable that the speed of the cell migration depends on the distance from the wound area. To verify whether this is the case, the filled area (i.e., the left and right side of the wound area) is divided into six local areas as shown in Fig. 8. These local areas were defined based on the distance from the wound area, from L1 to L3 at the left side and from R1 to R3 at the right side away from the wound area. The cells on the edges of the wound area migrate toward the center, therefore, these local areas also move toward the center with the time. For each local area, the average speed of the cell migration is computed as shown in Fig. 9. Understandably, the cells in condition A (control) move faster than the others in every local area, and the cells in condition $\mathrm{C}(100 \mathrm{nM})$ are the slowest. The graph indicates another interesting phenomenon in that the order of speeds are L1, L2 and L3 for the left side, and R1, R2 and R3 for the right side; that is, the speed of cell migration decreases with the distance from the wound edge.

3) Direction of the Cell Migration: To quantitatively analyze the direction of the cell migration, the distribution of cell migration directions on each condition was plotted by an angular histogram (rose diagram) as shown in Fig. 10. The first three rows show the distributions of the direction on each local area, respectively, for conditions A, B and C, and the bottom row shows the distributions on the whole areas left and right. We can observe that the cells tend to migrate toward the wound area in every local area on condition A and $\mathrm{B}$. The cell migrations in L1, R1 (nearest area to the wound) are most highly directional to the direction to the wound. Also the graphs indicate an interesting phenomenon that cell migration in condition $\mathrm{C}(100 \mathrm{nM})$ is less directed to the wound area $\left(0^{\circ}\right.$ for the left side, $180^{\circ}$ for the right side). This means that the speed of the cell migration toward the wound area is the slowest in condition C (Fig. 6 (d)) not because the migration speed is slow, but because the

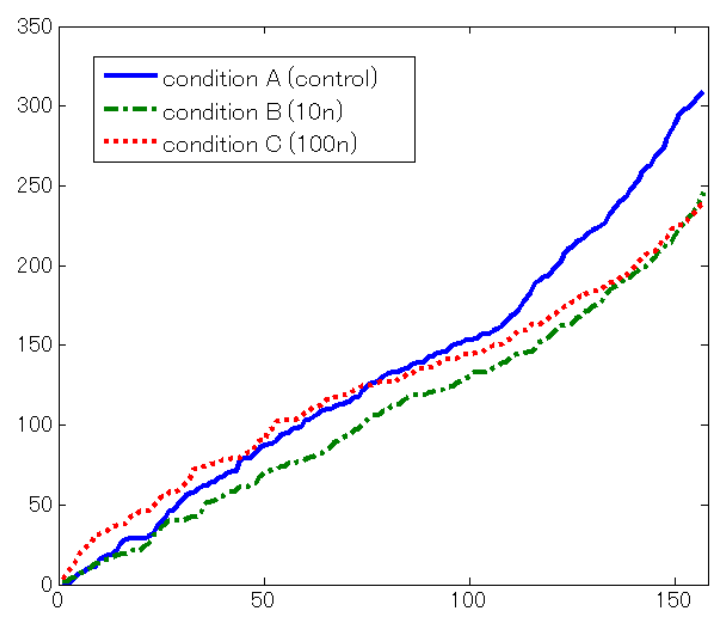

Fig. 12. The number of accumulated cell mitosis events at each frame.

migration direction is less directed.

4) Density Slope and Migration Direction: It is conceivable that the direction and speed of the cell migration depend on the density slope. For example, when the density slope is high, the cells on the area may move from the high density area to the low density area. When the density slope is small (i.e., the density is flat), the cells don't move much. To confirm the hypothesis, we compute the graph in which shows the relationship between density slope and migration direction as shown in Fig. 11. The horizontal axis indicates the cell density slope which is computed as how the cell density change over $x$ axis on Fig. 6. The illustration described below the graph indicates cell density on the two areas. As shown in the illustration, negative density slope indicates the density decreases from left to right direction on the area, positive density slope indicates the density increases from left to right direction on the area. The illustration described at the left side of the graph indicates the distribution of cell migration direction. As shown in the illustration, vertical axis indicates the directional migration speed i.e., how the cells move toward to the same direction. When the absolute value of the vertical axis is small, the cell migration on the area is less directed. When the absolute value of the vertical axis is high, the cell migration is highly directional to the direction from high density to the low density. Understandably, we can observe that the cell migration direction is highly directional on the area where the density slope is high, and migration direction is less directed on the area where the density slope is small.

5) Number of Cell Mitosis Events: To analyze how the number of cell mitosis events changes over time, we computed the number of accumulated mitosis events over the whole area. The results presented in Fig. 12 show that the number of mitosis events in the condition A (control) is higher than those in the other conditions. The curves of the accumulated number are almost linear. It indicates that the 


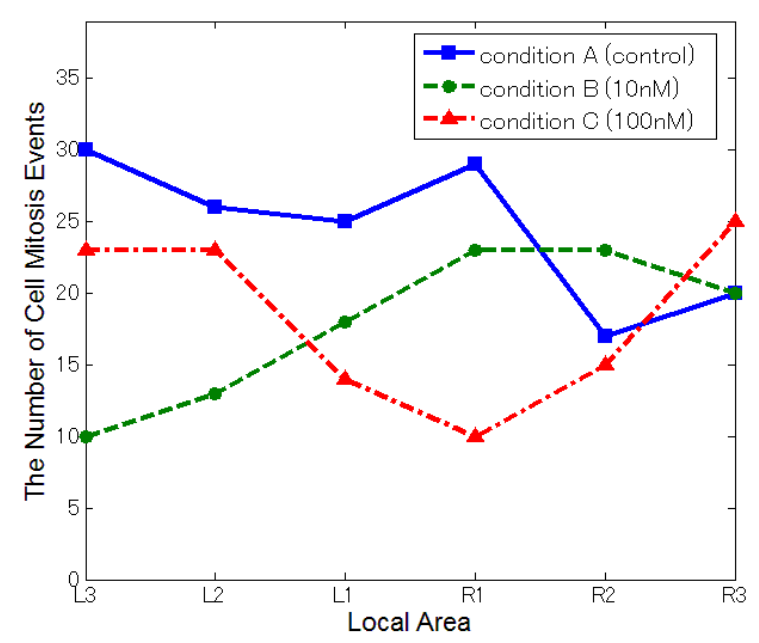

Fig. 13. The number of cell mitosis events at each local area.

mitosis events occur consistently.

To show how the number of the cell mitosis events changes by location, the number of the cell mitosis events is computed over each local area as shown in Fig. 13. In this graph, we observe no distinctive characteristics. This means that the culture condition has more effects to the number of mitosis events than the distance from the wound area does.

\section{Discussion AND CONCLUSION}

We presented the application of automatic cell tracking in phase-contrast microscopy images for wound healing assays in vitro in order to produce detailed quantitative analysis of the cell behaviors under three different culture conditions.

Understanding the cell behaviors is important to studying the influence of the environments including the types of medicines, the amount of the medicine, and materials in which these cells can grow. In the past, the simple measurement, such as the size of the wound area is often used, but such simple measures cannot provide the detailed analysis of cell behaviors. The spatio-temporal measurements of cell behaviors are important for critical analysis, because the cell culture conditions vary with time and space on the dish. Our system can provide such spatio-temporal cell behaviors measurements: the cell density, cell migration speed and direction, and statistics of cell mitosis events. The results of the experiments demonstrated the effectiveness of automatic cell tracking for quantitatively analyzing cell behaviors.

\section{ACKNOWLEDGMENTS}

We would like to thank cell tracking project members of Carnegie Mellon University: Dr. Sungeun Eom, Dr. Mei Chen, Elmer Ker, Dr. Phil Campbell and Dr. Lee Weiss. We really appreciate Dr. Naoki Yokoyama and Dr. Hideshi Hattori, who are working on Dai Nippon Printing, providing microscopy image sequences and a lot of comments about the cell behavior measurements to us.

\section{REFERENCES}

[1] D. House et al., "Tracking of Cell Populations to Understand their Spatio-Temporal Behavior in Response to Physical stimuli," CVPR Workshop MMBIA, 2009.

[2] D. Padfield et al., "Quantitative Biological Studies Enabled by Robust Cell Tracking," ISBI 2011.

[3] L. Chun-Chi et al., "In vitro scratch assay: a convenient and inexpensive method for analysis of cell migration in vitro," Nature Protocols, 2(2), 329-332, 2007

[4] C.J. MYarrow et al., "A high-throuput cell migration assay using scratch wound healing, a comparison of image-based readout methods," BMC Biotechnology, 4(21), 2004.

[5] S. Abbi et al., "Regulation of Focal Adhesion Kinase by a Novel Protein Inhibitor FIP200," Molecular Biology of the Cell, 13, 3178 3191, 2002.

[6] L.D. Nikolic et al., "Role of boundary conditions in an experimental model of epithelial wound healing," Am J Physical Cell Physiol, 291, 68-75, 2006.

[7] J.M. Zahm et al., "Cell Migration and Proliferation During the In Vitro Wound Repair of the Respiratory Epithelium," Cell Motility and the Cytoskeleton, 37, 33-43, 1997.

[8] T. Kanade et al., "Cell Image Analysis: Algorithms, System and Applications," IEEE WACV, 2011

[9] Z. Yin et al., "Understanding the Optics to Aid Microscopy Image Segmentation," MICCAI, 2010.

[10] S. Huh et al., "Automated mitosis detection of stem cell populations in phase-contrast microscopy images," IEEE Trans.Med. Imag.

[11] J.C. Yarrow et al., "Screening for Cell Migration Inhibitors via Automated Microscopy Reveals a Rho-Kinase Inhibitor," Chemistry \& Biology, 12, 385-395, 2005.

[12] R. Pril et al., "A high-content screen for inhibitors of cell migration in cancer metastasis using adenoviral knock-down," BTi April, 12, 385-395, 2009.

[13] C.B. Isenberg et al., "Vascular Smooth Muscle Cell Durotaxis Depends on Substrate Stiffness Gradient Strength,' Biopysical Jounal, 97, 1313$1322,2009$.

[14] M. Tamura et al., "Inhibition of Cell Migration, Spreading, and Focal Adhesions by Tumor Suppressor PTEN," Science, 280, 1614-1617, 1998.

[15] J. Gu et al., "Shc and FAK Differentially Regulate Cell Motility and Directionality Modulated by PTEN," Cell Biology, 146, 390-403, 1999. 